\title{
Arthrocnemo macrostachyi-Sarcocornietum hispanicae, a new halophytic plant community from eastern Iberian Península
}

\author{
Vicenta de la Fuente, Lourdes Rufo, Ana Teijeiro (*) \& \\ Daniel Sánchez-Mata (**)
}

\begin{abstract}
Fuente, V. de la, Rufo, L., Teijeiro, A. \& Sánchez-Mata, D. Arthrocnemo macrostachyi-Sarcocornietum hispanicae, a new halophytic plant community from eastern Iberian Península. Lazaroa 34: 267-272 (2013).

A new phytosociological association, Arthrocnemo macrostachyi-Sarcocornietum hispanicae, to frame the halophytic vegetation structured by Sarcocornia hispanica throughout saline habitats in eastern Iberian Peninsula is proposed. Some phytosociological remarks on Salicornietea fruticosae syntaxonomical typology are also given.
\end{abstract}

Keywords: Chenopodiaceae, Sarcocornia, Sarcocornia hispanica, Salicornietea fruticosae, halophytic vegetation.

Resumen: Fuente, V. de la, Rufo, L., Teijeiro, A. \& Sánchez-Mata, D. Arthrocnemo macrostachyi-Sarcocornietum hispanicae, una nueva asociación halofítica del este de la Península Ibérica. Lazaroa 34: 267-272 (2013).

Se propone la nueva asociación Arthrocnemo macrostachyi-Sarcocornietum hispanicae para englobar la vegetación halófila fruticosa del este de la Península Ibérica dominada por Sarcocornia hispanica. Además, se incluyen comentarios fitosociológicos sobre la tipología sintaxonómica de la clase Salicornietea fruticosae.

Palabras clave: Chenopodiaceae, Sarcocornia, Sarcocornia hispanica, Salicornietea fruticosae, vegetación halófila.

\section{INTRODUCTION}

New taxa are considered inside the Sarcocornia A.J. Scott genus (Chenopodiaceae) as a result of its recent taxonomic review in the Iberian Peninsula (DE LA FuENTE \& al., 2011, 2013). Specifically, the new species Sarcocornia hispanica Fuente, Rufo \& Sánchez-Mata have been recognized throughout eastern and south-eastern territories of the Iberian Peninsula.

Sarcocornia hispanica usually occurs in saline sandy depressions where usually it is not reached by sea water. In these habitats it can be accompanied mainly by Arthrocnemum macrostachyum. It could also be found far away from the coast in endorreic lakes derived from Tertiary materials, or even in canals of salt works. We know this taxon from xeric oceanic and pluviseasonal oceanic Mediterranean bioclimates, covering mesoand thermomediterranean thermotype territories with dry and semiarid ombrotype character (GIMÉnez LuQue \& Gómez Mercado, 2002; SAlaZAR \& al., 2002; AlONSO \& DE LA TORRE, 2002; Rivas-MARTíneZ \& al., 2007; SARIKA, 2012).

The complexes of plant communities structured by fruticose succulent chamaephytes in eastern Iberian Peninsula (Arthrocnemum macrostachyum, Sarcocornia fruticosa, Sarcocornia hispanica) are framed in the phytosociologial class Salicornietea fruticosae within tha alliances Salicornion fruticosae y Arthrocnemion glauci (RIVASMARTínez \& al., 2011).

\footnotetext{
* Departamento de Biología. Facultad de Ciencias. Universidad Autónoma de Madrid. Cantoblanco. E-28049 Madrid, Spain. E-mail: vicenta.fuente@uam.es, lourdes.rufo@uam.es, ana.teijeiro@estudiante.uam.es

** Departamento de Biología Vegetal II. Facultad de Farmacia. Universidad Complutense. E-28040 Madrid, Spain. E-mail: dsmata@farm.ucm.es
} 
Halophytic shrub communities structured by Arthrocnemum macrostachyum are widely distributed throughout Murcia-Almería biogeographical territories and developed on clay compacted soils; they are framed in the association Frankenio corymbosae-Arthrocnemetum macrostachyi defined for the coastal and mainland formations from Murcia and Alicante (RIVAS-MARTínEZ \& al., 1984).

The formations of fruticose succulent Sarcocornia from Murcia-Almería saline localities (previously only identified as Sarcocornia fruticosa) were included in different associations such as Salicornietum fruticosae Br.-Bl. 1928 (RIGUAL, 1972; Esteve, 1973), Cistancho phelypaeae-Sarcocornietum fruticosae Géhu ex Géhu \& GéhuFranck 1977 (AlCARAZ, 1984; AlCARAZ \& al., 1991; AlONSO, 1996; SALAZAR \& al., 2002) and finally, as the new proposed Limonio cossonianiSarcocornietum fruticosae Alonso \& de la Torre 2002. More recently LENDíNEZ \& al. (2012:232) published the new association Limonio majorisSarcocornietum fruticosae Lendínez, Marchal \& Salazar 2012 to include this formations from the Baza and surroundings saline territories (Granada).

The recognition of the new taxon Sarcocornia hispanica by DE LA FUENTE \& al. (2011) distributed throughout the semiarid saline areas from the eastern and south-eastern Iberian Peninsula forces a precise revision of the shrubby halophytic vegetation structured by fruticose succulent Chenopodiaceae.

Our recent studies concerning the halophytic vegetation of these territories allow us to recognize and propose a new association structured by Sarcocornia hispanica and a nomenclatural correction.

\section{DATA AND METHODS}

Our vegetation study follows the usual phytosociological methods. We took relevés following the Braun-Blanquet method (BRAUN-BLANQUET, 1979).

Biogeographical and bioclimatical concepts follows the proposals of RIVAS-MARTínEZ \& al. (2007). Phytosociological nomenclature follows the contributions and proposals of RIVAS-MARTíNEZ \& al. $(2001,2002,2011)$ and the current issue of the International Code of Phytosociological Nomenclature (ICPN, WEBER \& al., 2000).

For taxonomical nomenclature we follow, except if the authority is indicated, Flora Europaea (TuTin \& al., 1964-1993), the EuroMed Database (2006), and the published volumes of Flora iberica (CASTROVIEJo \& al., 1986-2010)

\section{RESULTS AND SYNTAXONOMICAL DISCUSSION}

SAlicornietea Fruticosae Br.-Bl. \& Tüxen ex A. \& O. Bolòs 1950

Vegetation developed on swamps and marine estuaries, and inland saline areas. This phytosociological class frames the shrubby halophytic plant communities distributed throughout European coastal and inland saline territories.

\section{Salicornietalia fruticosae Br.-Bl. 1933}

Vegetation developed on coastal areas influenced by sea tides and inland saline habitats.

\section{Salicornion fruticosae Br.-Bl. 1933}

Fruticose and suffruticose saline vegetation developed throughout infra-mesomediterranean and thermo-mesotemperate thermotype territories in areas influenced by sea tides.

Sarcocornienion fruticosae Rivas-Martínez \& Costa in Rivas-Martínez \& al. 2011

Halophytic shrubby vegetation structured by Sarcocornia fruticosa. We recognize two associations throughout eastern and south-eastern saline areas of Iberian Peninsula:

\section{Statico bellidifoliae-Salicornietum fruticosae Br.-B1. 1933}

Association widely distributed on coastal saline habitats of Catalonia-Provence-Balearic Islands biogeographical territories with thermo-mesomediterranean thermotype character and structured by 
Sarcocornia fruticosa. These halophytic vegetation grows on coastal saline areas and dune depressions supporting temporary ponding and summer drought.

COSTA \& BOIRA (1981) described within this association two subassociations: halimietosum portulacoidis, for silty loam soil texture and sporoboletosum pungentis for sandy loam soil texture. Table 1 shows an own compilation of non-published relevés made on Catalonia coastal territories.

\section{Limonio cossoniani-Sarcocornietum fruticosae} Alonso \& de la Torre 2002

Association widely distributed on coastal saline habitats of Murcia-Almería biogeographical territories with thermo-mesomediterranean thermotype character and structured by Sarcocornia fruticosa. Other frequent species within this vege- tation-type are the succulent halophytes Arthrocnemum macrostachyum and Limbarda crithmoides; the rosulate hemicriptophytes Limonium cossonianum, Limonium caesium, and Limonium santapolense, and other halophytes such as Halimione portulacoides or Frankenia corymbosa. Sarcocornia hispanica can be very occasional in this halophytic formations. Table 2 shows an own compilation of non-published relevés made on eastern coastal Iberian Peninsula territories (Murcia-Almería biogeographical territories).

In the original diagnosis of this association Alonso \& De LA ToRre (2002) included the saline habitats of Villena, Granada, Murcia and Alicante. However, in these territories we recognize Sarcocornia hispanica. Limonio cossoniani-Sarcocornietum fruticosae is restricted to coastal saline habitats of Murcia-Almería biogeographical territories.

Table 1

Statico bellidifolii-Salicornietum fruticosae Br.B1. 1933 salicornietosum fruticosae 1-7

halimionetosum portulacoidis 8-18

(Sarcocornienion, Salicornion fruticosae, Salicornietalia fruticosae, Salicornietea fruticosae)

\begin{tabular}{|c|c|c|c|c|c|c|c|c|c|c|c|c|c|c|c|c|c|c|}
\hline $\begin{array}{l}\text { Area }\left(\mathrm{m}^{2}\right) \\
\text { Cover }(\%)\end{array}$ & $\begin{array}{c}20 \\
100 \\
1\end{array}$ & $\begin{array}{c}50 \\
100 \\
2\end{array}$ & $\begin{array}{c}20 \\
100 \\
3\end{array}$ & $\begin{array}{l}30 \\
80 \\
4\end{array}$ & $\begin{array}{c}20 \\
80 \\
5\end{array}$ & $\begin{array}{c}10 \\
100 \\
6\end{array}$ & $\begin{array}{r}20 \\
80 \\
7\end{array}$ & $\begin{array}{c}20 \\
80 \\
8\end{array}$ & $\begin{array}{c}20 \\
100 \\
9\end{array}$ & $\begin{array}{l}50 \\
80 \\
10\end{array}$ & $\begin{array}{c}20 \\
100 \\
11\end{array}$ & $\begin{array}{c}20 \\
100 \\
12\end{array}$ & $\begin{array}{c}20 \\
100 \\
13\end{array}$ & $\begin{array}{l}50 \\
80 \\
14\end{array}$ & $\begin{array}{l}50 \\
80 \\
15\end{array}$ & $\begin{array}{c}20 \\
100 \\
16\end{array}$ & $\begin{array}{c}20 \\
100 \\
17\end{array}$ & $\begin{array}{c}20 \\
100 \\
18\end{array}$ \\
\hline & & & & & & & & & & & & & & & & & & \\
\hline \multicolumn{19}{|l|}{ Characteristics } \\
\hline Sarcocornia fruticosa & 5 & 5 & 4 & 5 & 4 & 4 & 5 & 4 & 5 & 3 & 5 & 5 & 4 & 4 & 3 & 4 & 4 & 4 \\
\hline Limbarda crithmoides & . & . & . & . & 1 & . & 1 & . & . & . & . & + & + & . & . & 2 & 2 & 1 \\
\hline Arthrocnemum macrostachyum & . & . & + & . & 1 & 1 & . & . & . & . & 1 & . & . & 2 & + & . & . & \\
\hline Suaeda vera & . & . & . & . & . & . & . & . & . & . & . & . & . & 1 & . & 1 & + & \\
\hline $\begin{array}{l}\text { Limonium angustebracteatum } \\
\text { Differentials of halimionetosum }\end{array}$ & . & . & . & . & . & . & . & . & . & . & 2 & . & . & + & . & . & . & \\
\hline $\begin{array}{l}\text { Halimione portulacoides } \\
\text { Companions }\end{array}$ & . & . & . & . & . & . & . & 1 & + & 1 & 2 & 2 & 3 & + & 1 & 3 & 3 & 3 \\
\hline Phragmites australis & 1 & 1 & 1 & 1 & 1 & 1 & . & . & + & . & . & . & . & . & . & 1 & . & . \\
\hline Juncus acutus & . & 1 & 1 & + & . & . & 2 & . & . & . & . & . & . & . & . & $\cdot$ & . & \\
\hline Juncus maritimus & . & . & . & . & 2 & 2 & . & . & . & . & . & . & 1 & . & . & . & . & 1 \\
\hline Elytrigia juncea & . & . & . & + & . & . & 1 & . & + & . & . & . & . & . & . & . & . & 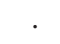 \\
\hline Limonium virgatum & . & . & . & . & . & + & . & . & . & 1 & . & . & . & . & 1 & . & . & \\
\hline Juncus subulatus & . & + & . & . & . & . & 1 & . & . & . & . & . & . & . & $\cdot$ & . & . & \\
\hline Salicornia ramosissima & . & . & . & . & . & . & . & 1 & . & . & . & . & . & . & 1 & . & . & \\
\hline
\end{tabular}

Other species: characteristics: Limonium dufourii + in 6; Limonium girardianum + in 11; Limonium bellidifolium + in 13; Puccinellia festuciformis 1 in 17; Limonium densissimum + in 18. Companions: Suaeda splendens + in 5; Polygonum equisetiforme + in 7.

Localities: 1, 4, 9. Gola del Ter, L'Estartit, Gerona, 31TEG1857; 2, 3, 7, 10, 15. Parque Natural Cabanes, Torreblanca, Castellón, 31TBE6354; 5, 6. El Saler, Valencia, 30SYJ3163; 6, 11, 14. Estanys d'Almenara, Almenara, Castellón, 31SYK4103; 8, 13, 16, 18. San Carlos de la Rápita, Tarragona, 31TCF2108; 12, 17. Parque Natural Aiguamolls de l'Empordà, Sant Pere Pescador, Gerona, 31TEG0972. 
Table 2

Limonio cossoniani-Sarcocornietum fruticosae Alonso \& de la Torre 2000

(Sarcocornienion, Salicornion fruticosae, Salicornietalia fruticosae, Salicornietea fruticosae)

\begin{tabular}{lcccccccccccccc}
\hline Area $\left(\mathrm{m}^{2}\right)$ & 50 & 50 & 50 & 20 & 20 & 20 & 20 & 20 & 30 & 20 & 20 & 20 & 20 & 30 \\
Cover $(\%)$ & 100 & 100 & 80 & 100 & 80 & 100 & 80 & 80 & 80 & 80 & 90 & 100 & 100 & 80 \\
Relevé N. & 1 & 2 & 3 & 4 & 5 & 6 & 7 & 8 & 9 & 10 & 11 & 12 & 13 & 14 \\
\hline
\end{tabular}

Characteristics

Sarcocornia fruticosa

Arthrocnemum macrostachyum

Halimione portulacoides

Limonium cossonianum

Limbarda crithmoides

Limonium caesium

Companions

Salicornia ramosissima

Phragmites australis

Juncus maritimus

Lygeum spartum

\begin{tabular}{|c|c|c|c|c|c|c|c|c|c|c|c|c|}
\hline 4 & 4 & 3 & 5 & 4 & 4 & 4 & 4 & 5 & 4 & 4 & 4 & 4 \\
\hline & 1 & 1 & 2 & 2 & 1 & 1 & 1 & 2 & 1 & 1 & 2 & 2 \\
\hline & 2 & . & 1 & . & 1 & . & . & 2 & . & 2 & . & 2 \\
\hline . & . & . & . & . & . & 2 & . & 1 & . & . & 3 & 2 \\
\hline . & . & $\cdot$ & . & . & 1 & . & . & . & . & . & . & 1 \\
\hline . & . & . & . & . & . & . & . & $\cdot$ & . & 2 & . & . \\
\hline 2 & . & 1 & . & . & . & . & + & . & . & . & . & • \\
\hline . & . & . & . & 1 & . & 1 & 1 & . & + & . & . & . \\
\hline - & . & $\cdot$ & . & . & . & . & 2 & . & . & - & 2 & • \\
\hline & . & . & . & . & . & . & . & . & . & . & + & . \\
\hline
\end{tabular}

Other species: characteristics: Frankenia corymbosa + y Limonium santapolense 2 in 10; Sarcocornia hispanica + in 13, Suaeda vera 1 in 14. Companions: Suaeda spicata 1 in 10; Mesembryanthemum crystallinum, Mesembryanthemum nodiflorum and Parapholis incurva + in 12; Juncus acutus + in 14.

Localities: 1, 5, 12. Torrevieja, Laguna de la Mata, Alicante, 30SYH0212; 2, 4, 6. Lopagán, San Pedro del Pinatar, Murcia, 30SXG9791; 3, 8, 9, 11, 14. Torrevieja, Laguna Grande, Alicante, 30SXH9809; 7, 13. Salinas de Sta. Pola, Alicante, 30SYH1029; 10. Torre Tamarit, Santa Pola, Alicante, 30SYH0827.

Arthrocnemion glauci Rivas-Martínez \& Costa 1984

Fruticose and suffruticose saline vegetation developed throughout infra-supramediterranean inland territories or in areas not influenced by sea tides and structured by Arthrocnmemum macrostachyum.

Arthrocnemenion macrostachyi (Rivas-Martínez \& Costa 1984) Rivas-Martínez \& Costa in RivasMartínez \& al. 2011

Halophytic vegetation structured by the succulent chamaephytic halophyte Arthrocnmemum macrostachyum. We include in this phytosociological unit the halophytic formations dominated by the iberian halophyte Sarcocornia hispanica:

\section{Limonio majoris-Sarcocornietum hispanicae} Lendínez, Marchal \& Salazar 2012 nom. corr. hoc loco (ICPN, art. 43)

[sub Limonio majoris-Sarcocornietum fruticosae Lendínez, Marchal \& Salazar 2012 (in Lagascalia 32: 232)]
Endemic association known only from the saline habitats of Guadix-Baza biogeographical territories (Granada). This association frames the shrubby halophytic vegetation developed on endorheic depressions temporarily flooded in winter and spring and dry up in summer.

The nomenclatural correction is obliged strictly following the current issue of the ICPN (WEBER \& al., 2000) because of the recognized taxon in the area of this association is Sarcocornia hispanica (DE LA FuENTE \& al., 2011), not Sarcocornia fruticosa as published by LENDíNEZ \& al. (2012).

Arthrocnemo macrostachyi-Sarcocornietum hispanicae ass. nova hoc loco (Table 3; holotypus, rel. 4)

Table 3 shows nine relevés supporting this phytosociological association proposed here as new and structured by the succulent halophyte Sarcocornia hispanica. As frequent species we can remark other shrubby halophytes such as Arthrocnemum macrostachyum, Suaeda vera or 
Table 3

Arthrocnemo macrostachyi-Sarcocornietum hispanicae ass. nova

(Arthrocnemenion macrostachyi, Arhtrocnemion glauci, Salicornietalia, Salicornietea fruticosae)

\begin{tabular}{|c|c|c|c|c|c|c|c|c|c|}
\hline Altitude (m) & 5 & 10 & 10 & 520 & 520 & 5 & 10 & 560 & 150 \\
\hline Area $\left(\mathrm{m}^{2}\right)$ & 40 & 50 & 50 & 20 & 20 & 50 & 20 & 30 & 20 \\
\hline Cover $(\%)$ & 80 & 100 & 100 & 100 & 80 & 80 & 80 & 100 & 80 \\
\hline Relevé number & 1 & 2 & 3 & 4 & 5 & 6 & 7 & 8 & 9 \\
\hline \multicolumn{10}{|l|}{ Characteristics } \\
\hline Sarcocornia hispanica & 4 & 5 & 4 & 4 & 4 & 1 & 4 & 4 & 4 \\
\hline Suaeda vera & . & + & 1 & + & + & . & + & 2 & + \\
\hline Limonium cossonianum & . & + & . & . & . & 1 & 1 & 1 & 2 \\
\hline Arthrocnemum macrostachyum & . & . & 1 & 2 & + & 3 & . & . & + \\
\hline Halimione portulacoides & . & . & . & 1 & 1 & 1 & 1 & . & 1 \\
\hline \multicolumn{10}{|l|}{ Companions } \\
\hline Phragmites australis & 2 & + & 1 & . & . & . & . & 1 & + \\
\hline
\end{tabular}

Other species: Characteristics: Limbarda crithmoides 1 in 1; Sarcocornia fruticosa 2 in 6; Limonium caesium + in 8; Limonium angustebracteatum 1 in 9. Companions: Lygeum spartum 1 in 6; Mesembryanthemum crystallinum and Suaeda spicata + in 7; Elytrigia curvifolia 1 and Sphenopus divaricatus + in 8.

Localities: 1. Salinas de Santa Pola, Alicante, 30SYH1029; 2, 3, 7. Laguna del Hondo, San Felipe Neri, Alicante, 30SXH9728; 4, 5. Las Virtudes, Alicante, 30SXH8075; 6. Laguna Grande, Torrevieja, Alicante, 30SXH9809; 8. Cordovilla, Albacete, 30SXH2070; 9. Rambla del Ajauque, La Fortuna, Murcia, 30SXH6527. Holotypus ass., rel. 4.

Halimione portulacoides; frequent rosulate hemicriptophytes are Limonium angustebracteatum, Limonium caesium, Limonium cossonianum and Limonium eugeniae. Phragmites australis, Juncus maritimus and Juncus subulatus detected brackish water.

The biogeographical area of this association cover inland saline areas of La Mancha-Murcia and Murcia-Almería biogeographical territories. This vegetation-type grows on inland saline depressions or endorheic lagoons developed on Tertiary geologic materials; also it can grows on channels of coastal saline explotations. The ve- getational contacts in coastal saline habitats are to the wettest area with the communities structured by Sarcocornia fruticosa (Limonio cossoniani-Sarcocornietum fruticosae); to the more saline habitats with the communities structured by Arhtrocnemum macrostachyum (Frankenio corymbosae-Arhtrocnemetum macrostachyi); to the anthropogenic sites with the communities structured by Suaeda vera (Frankenio corymbosae-Suaedetum verae) and to the driest areas with the arid-dry communities structured by Lygeum spartum (Lygeo sparti-Limonion furfuracei, $\mathrm{Li}$ monietalia).

\section{SYNTAXONOMICAL TYPOLOGY}

SALICORNIETEA FRUTICOSAE Br.-Bl. \& Tüxen ex A. \& O. Bolòs 1950

Salicornietalia fruticosae $\mathrm{Br}$.-B1. 1933

Salicornion fruticosae $\mathrm{Br}$-Bl. 1933

Sarcocornienion fruticosae Rivas-Martínez \& Costa in Rivas-Martínez \& al. 2011

Statico bellidifoliae-Salicorcornietum fruticosae Br.Bl. 1933

Limonio cossoniani-Sarcocornietum fruticosae Alonso \& de la Torre 200

Arthrocnemion glauci Rivas-Martínez \& Costa 1984

Arthrocnemenion macrostachyi (Rivas-Martínez \& Costa 1984) Rivas-Martínez \& Costa 2011

Limonio majoris-Sarcocornietum hispanicae Lendínez, Marchal \& Salazar 2012 nom. corr.

Fuente \& al. 2013

Arthrocnemo macrostachyi-Sarcocornietum hispanicae ass. nova 


\section{REFERENCES}

Alcaraz, F. - 1984- Flora y vegetación del NE de Murcia - Publ. Univ. Murcia. Murcia.

Alcaraz, F., Díaz, T.E., Rivas-Martínez, S. \& SánchezGómez, P. - 1989- Datos sobre la vegetación del sureste de España: provincia biogeográfica murciano-almeriense - Itinera Geobot. 2: 2-133.

Alcaraz, F., Sánchez, P., Torre, A. de la, Ríos, S. \& Alvarez, J. - 1991 - Datos sobre la vegetación de Murcia (España). Guía geobotánica de la Excursión de las IX Jornadas de Fitosociología - Publ. Univ. Murcia. 162 pp. Murcia.

Alonso, M.A. - 1996 - Flora y Vegetación del Valle de Villena (Alicante) - Publ. Inst. Cultura Juan Gil-Albert, Textos Univ. Dip. Prov., Alicante.

Alonso, M.A. \& de la Torre, A. - 2002- Datos sobre las comunidades de Sarcocornia fruticosa (L.) A.J. Scott en la provincia murciano-almeriense (SE de España) Acta Bot. Malacitana 27: 288-294.

Bolòs, A. de \& Bolòs, O. de - 1950 - Vegetación de las comarcas barcelonesas - Publ. Inst. Esp. Est. Medit., Barcelona.

Br.-Bl, J. - 1933 - Prodrome des groupements végétaux, fasc. I (Ammophiletalia et Salicornietalia médit.) Comm. Int. des Prodr. Phytosociol. 23 pp. Montpellier.

Braun-Blanquet, J. - 1979- Fitosociología - Ed. Blume, Madrid. 820 pp.

Castroviejo, S. \& al. (Eds.) - 1986-2010 - Flora iberica. Plantas vasculares de la Península Ibérica e Islas Baleares - R. Jard. Bot. CSIC, Madrid.

Costa, M. \& Boira, H. - 1981 - La vegetación costera valenciana: los saladares - An. Jard. Bot. Madrid 38(1): 233-244.

Esteve, F. - 1973 - Vegetación y flora de las regiones central y meridional de la provincia de Murcia - Publ. Centr. Edaf. Biol. Apl. Segura, Murcia. 451 pp.

Euro+Med - 2006 - Euro+Med PlantBase - The information resource for Euro-Mediterranean plant diversity Publ. Internet http://ww2.bgbm.org/EuroPlusMed/

Fuente, V. de la, Rufo, L. \& Sánchez-Mata, D. -2011Sarcocornia hispanica (Chenopodiaceae), a new species from the Iberian Peninsula - Lazaroa 32: 9-13.

Fuente, V. de la, Oggerin, M., Rufo, L., Rodríguez, N., Ortuñez, E., Sánchez-Mata, D. \& Amils, R. -2013- A micromorphological and phylogenetic study of Sarco- cornia A.J. Scott (Chenopodiaceae) on the Iberian Peninsula - Plant Biosys. 147(1): 158-173.

Giménez Luque, E. \& Gómez Mercado, F. -2002 - Análisis de la flora vascular de la Sierra de Gádor (Almería, España) - Lazaroa 23: 35-43.

Lendínez, M.L., Marchal, F.M. \& Salazar, C. -2012- Una nueva asociación de vegetación halófila en el sureste de la Península Ibérica (España): Limonio majoris-Sarcocornietum fruticosae - Lagascalia 32: 229-236.

Rigual, A, - 1972 - Flora y vegetación de la provincia de Alicante (El paisaje vegetal alicantino) - Publ. Inst. Estud. Alicantinos 2(1): 1-403.

Rivas-Martínez, S., Alcaraz, F., Belmonte, D., Cantó, P., Sánchez-Mata, D. -1984-Contribución al conocimiento de la vegetación de los saladares del sureste de la Península Ibérica (Arhtrocnemion glauci) - Doc. Phytosoc. 8: 335-340.

Rivas-Martínez, S., Díaz, T.E., Fernández-González, F., Izco, J., Loidi, J., Lousã, M. \& Penas, A. -2002 - Vascular plant communities of Spain and Portugal. Addenda to the syntaxonomical checklist of 2001 - Itinera Geobot. 15(1): 5-432.

Rivas-Martínez, S., Fernández-González, F., Loidi, J., Lousã, M. \& Penas, A. -2001 - Syntaxonomical Checklist of Vascular Plant Communities of Spain and Portugal to association level - Itinera Geobot. 14: 1-341.

Rivas-Martínez S. \& al. -2007 - Mapa de series, geoseries y geopermaseries de vegetación de España. Parte I Itinera Geobot. 17: 5-436.

Rivas-Martínez S. \& al. -2011 - Mapa de series, geoseries y geopermaseries de vegetación de España. Parte II Itinera Geobot. 18(1-2): 5-800.

Salazar, C., Torres, J.A., Marchal, F.M., Cano, E. -2002La vegetación edafohigrófila del distrito GuadicianoBastetano (Granada-Jaén, España) - Lazaroa 23: 4564.

Sarika, M. - 2012 - Notes on the flora and vegetation of some coastal ecosystems of Sterea Ellas and eastern continental Greece (Greece) - Lazaroa 33: 65-99.

Tutin, T. G. \& al. (Eds.) - 1964-1993 - Flora Europaea Cambridge Univ. Press, Cambridge.

Weber, H.E., Moravec, J. \& Theurillat, J.P. - 2000 - International Code of Phytosociological Nomenclature, 3rd ed. - J. Veg. Sci. 11: 739-768. 